Textures and Microstructures, 1988, Vol. 10, pp. 21-35

Reprints available directly from the publisher

Photocopying permitted by license only

(C) 1988 Gordon and Breach Science Publishers Inc.

Printed in the United Kingdom

\title{
A Positivity Method for the Determination of Complete Orientation Distribution Functions
}

\author{
M. DAHMS \\ GKSS-Forschungszentrum, Max-Planck-Straße, D-2054 Geesthacht \\ and \\ H. J. BUNGE \\ Institut für Metallkunde und Metallphysik, Großer Bruch 23, D-3392 Clausthal- \\ Zellerfeld
}

(Received 15 December, 1987)

A refinement of the zero-range method, a procedure to calculate the odd order coefficients in the series expansion method of texture analysis, is presented. The only assumption in this procedure is the positivity condition. In this respect, it is comparable to the quadratic method. Contrary to this method, however, the even order coefficients are not changed. No zero range in the pole figures and no shape of the existing texture is to be assumed.

KEY WORDS: Complete ODF, Iterative procedure, Positive solution, Ambiguity of the solution, Titanium, Calcite.

\section{INTRODUCTION}

Because of Friedel's law, an experimental pole figure is always a superposition of the $(h k l)$ and $(\bar{h} \bar{k} \bar{l})$ Bragg reflections, which leads to the effect that in the series expansion method (Bunge (1982)) only the even order coefficients of the orientation distribution function (ODF) can be calculated directly. 
The so obtained even or reduced ODF contains besides the peaks representing the measured data some (positive or negative) false peaks known as ghosts (Matthies (1979)). Three methods to determine the unknown odd order coefficients have been published thus far.

- the zero-range method (Bunge and Esling (1979))

- the model-function method (Lücke et al. (1981))

- the quadratic method (VanHoutte (1983))

They all suffer from some restrictions. When using model functions the solution depends on the introduced model. In the quadratic method big systems of linear equations are to be solved in an iterative procedure which requires much computer time and memory space. In this method, also the even part is modified when determining the odd part. The zero-range method requires an assumption of the zero range in the pole figures. It has been shown by Lee (1984), Lee et al. (1986) and Welch et al. (1987) that the odd order coefficients are not very sensitive to the choice of the zero-range. Thus, this method will be the base of the presented refined method.

\section{MATHEMATICAL FUNDAMENTALS}

The unknown complete ODF, $f(g)$, can be written as a sum of even and odd order functions

$$
f(g)=\tilde{f}(g)+\tilde{f}(g) \geq 0
$$

If we define the zero range by

$$
\mathbf{Z}_{0}=\{g \mid f(g)=0\}
$$

it is valid for $g \in \mathbf{Z}_{0}$ that

$$
\tilde{f}(g)=-\tilde{f}(g)
$$

Since the condition fixes $\tilde{f}$ only in the zero range, a great variety of functions $\tilde{\tilde{f}}$ is still possible. Their choice is only restricted by Eq. (1) and the condition

$$
\oint \tilde{f}(g) d g=0
$$




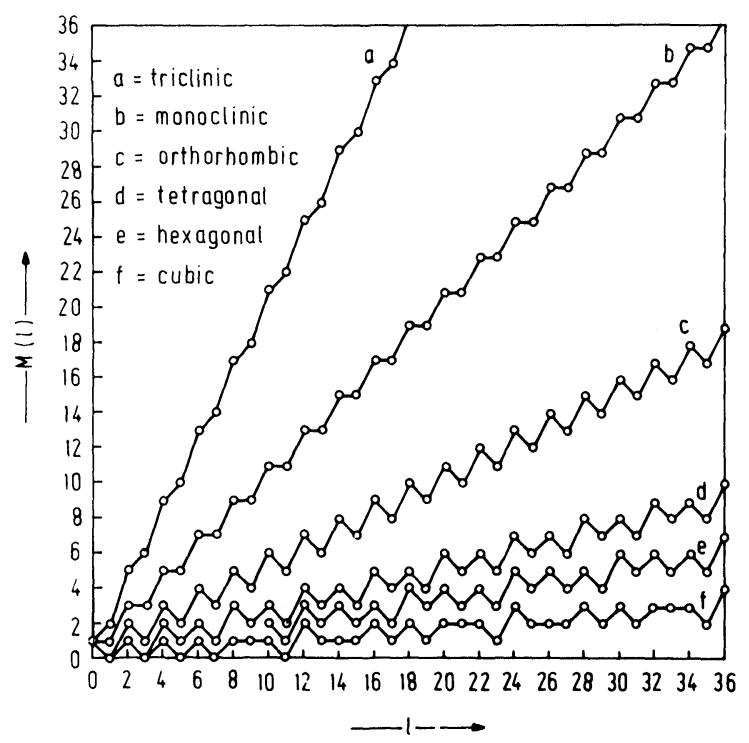

Figure 1 Number of linearly independent spherical harmonics of different symmetries as a function of the degree of series expansion.

In the series expansion method, the ODF, $f(g)$, is represented by a series of generalized spherical harmonics $\dot{T}_{l}^{\mu \nu}$ with coefficients $C_{l}^{\mu \nu}$, where (:) and (.) denote crystal and sample symmetry, respectively. The ODF is written

$$
f(g)=\sum_{l=0}^{L} \sum_{\mu=1}^{M(l)} \sum_{v=1}^{N(l)} C_{l}^{\mu v} \dot{T}_{l}^{\mu v}(g)
$$

The even and odd ODF can be obtained by just omitting the odd and even order coefficients, respectively. $M(l)$ and $N(l)$ depend on crystal and sample symmetry, respectively. They are shown in Figure 1.

If the even ODF is given up to a certain degree of series expansion $L$, an approximation to the odd ODF can be found by solving a least squares problem

$$
\int_{\mathbf{z}_{0}}[\tilde{f}(g)+\tilde{f}(g)]^{2} d g=\min
$$




\begin{tabular}{lr} 
Table 1 Number of unknown \\
odd order coefficients $\left(L_{1}=23\right)$ \\
$\begin{array}{l}\text { with orthorhombic sample sym- } \\
\text { metry depending on crystal } \\
\text { symmetry }\end{array}$ \\
\hline crystal symmetry & $I$ \\
\hline cubic & 65 \\
hexagonal & 144 \\
tetragonal & 235 \\
trigonal & 336 \\
orthorhombic & 506 \\
monoclinic & 1078 \\
\hline
\end{tabular}

Combination of Eq. (5) and Eq. (6) and setting the derivative with respect to $l^{\prime}, \mu^{\prime}$ and $v^{\prime}$ to zero leads to

$$
\sum_{l=1(2)}^{L_{1}} \sum_{\mu=1}^{M(l)} \sum_{v=1}^{N(l)} C_{l}^{\mu v} \cdot \int_{\mathbf{Z}_{0}} \tilde{T}_{l}^{\mu v}(g) \dot{\tilde{T}}_{l^{\prime}}^{\mu^{\prime} v^{\prime}}(g) d g=\int_{\mathbf{Z}_{0}} \tilde{f}(g) \dot{\tilde{T}}_{l}^{\mu v}(g) d g
$$

This is a system of linear equations with

$$
I=\sum_{l=1(2)}^{L_{1}} M(l) \cdot N(l)
$$

unknowns. The number $I$ is shown in Table 1 for different crystal symmetries and orthorhombic sample symmetry and $L_{1}=23$.

It is seen that, especially for lower symmetries, the system of linear equations becomes large. Rounding errors may become serious, such that iteration procedures are necessary to reduce these errors. Additionally, the zero range has to be determined somehow. This has been done in several publications (Lee (1984), Lee et al. (1986) and Welch et al. (1987)) by projecting the zero ranges of the pole figures into the orientation space. Thereby the problem arises how to define a physical zero range, because quite often no pole figure contains a mathematical zero range. This problem can be avoided by using a different approach, which will be described in the following.

The $n$th approximation of the complete ODF ${ }^{(n)} f$ may be defined as a sum of the $n-1$ st approximation of the complete $\operatorname{ODF}^{(n-1)} f$ 
and the $n$th correction term of the odd $\operatorname{ODF}^{(n)} \tilde{\tilde{f}}$. As a zero order approximation, the even ODF is taken.

$$
{ }^{(n)} f(g)={ }^{(n-1)} f(g)+{ }^{(n)} \tilde{\tilde{f}}(g), \quad{ }^{(0)} f(g)=\tilde{f}(g)
$$

If we define the negative range of the $n$th approximation of the complete ODF

$$
{ }^{(n)} \mathbf{Z}_{-}=\left\{\left.g\right|^{(n)} f(g)<0\right\}
$$

an estimate of the correction term can be obtained. For $g \epsilon^{(n-1)} \mathbf{Z}_{-}$ it may be set

$$
{ }^{(n)} \hat{f}(g)=-{ }^{(n-1)} f(g)
$$

and for $g \notin^{(n-1)} \mathbf{Z}_{-}$

$$
{ }^{(n)} \hat{f}(g)=0
$$

The determination of ${ }^{(n)} \tilde{\tilde{f}}$ is then carried out in such a way that negative ranges of the $n-1$ st approximation of the complete ODF should be brought to zero and positive ranges are kept unchanged. The unknown function ${ }^{(n)} \tilde{\tilde{f}}$ is to be approximated by a series of odd order, which must fulfil the minimization condition:

$$
\oint\left[{ }^{(n)} \hat{f}(g)-{ }^{(n)} \tilde{f}(g)\right]^{2} d g=\min
$$

with

$$
{ }^{(n)} \tilde{\tilde{f}}(g)=\sum_{l=1(2)}^{L_{1}} \sum_{\mu=1}^{M(l)} \sum_{v=1}^{N(l)}(n) \tilde{\tilde{C}}_{l}^{\mu v} \dot{T}_{l}^{\mu v}(g)
$$

This leads to:

$$
\sum_{l=1(2)}^{L} \sum_{\mu=1}^{M(l)} \sum_{v=1}^{N(l)}{ }^{(n)} \tilde{C}_{l}^{\mu \nu} \cdot \oint \dot{T}_{l}^{\mu \nu}(g) \dot{T}_{l^{\prime}}^{\mu^{\prime} v^{\prime}}(g) d g=\oint(n) \hat{f}(g) \dot{T}_{l}^{\mu v}(g) d g
$$

The main difference of Eq. (15) to Eq. (7) is the fact that the integrals are to be taken over the whole orientation space. Using the orthogonality relation

$$
\oint \dot{T}_{l}^{\mu v}(g) \dot{T}_{l^{\prime}}^{\mu^{\prime} v^{\prime}} d g= \begin{cases}0 & v \neq v^{\prime} \vee \mu \neq \mu^{\prime} \vee l \neq l^{\prime} \\ \frac{8 \pi^{2}}{2 l+1} & v=v^{\prime} \wedge \mu=\mu^{\prime} \wedge l=l^{\prime}\end{cases}
$$


Eq. (15) is simplified to

$$
{ }^{(n)} \tilde{C}_{l}^{\mu v}=\frac{2 l+1}{8 \pi^{2}} \cdot \oint(n) \hat{f}(g) \dot{T}_{l}^{\mu v}(g) d g
$$

Thus, by integrating over the whole orientation space, each coefficient can be determined independently. Additionally, the result is stable, because no strong negative values of the odd ODF outside the negative range of ${ }^{(n-1)} f(g)$ may occur.

The function ${ }^{(n)} f(g)$ surely has still negative ranges, from which a higher order approximation of the odd order coefficients can be determined and so forth. The final solution of the odd order coefficients is a summation over all iteration steps.

$$
\tilde{C}_{l}^{\mu v}=\sum_{n}{ }^{(n)} \tilde{C}_{l}^{\mu v}
$$

The iteration has to be truncated somewhere. The highest negative value of the complete ODF can be used as truncation criterion. If a certain value is reached, the iteration may be stopped. Also an integral criterion is possible, where the integral over the negative range of the complete ODF is calculated.

$$
{ }^{(n)} S_{-}=\int_{(n)}{ }_{\mathbf{Z}_{-}}{ }^{(n)} f(g) d g
$$

In practice, this integral is replaced by a weighted sum of negative intensities, e.g. calculated in steps of $5^{\circ}$ through the Euler space (Welch et al. (1987)).

If there is no negative range in the even ODF, Eq. (11-18) lead to trivial zero coefficients $\tilde{C}_{l}^{\mu \nu}$. This illustrates the fact that the odd part of the texture function is, in general, not uniquely defined by the pole figures (even if an infinite number of pole figures were available). The odd part $\tilde{\tilde{f}}(g)$ of the texture function is only restricted by the positivity condition Eq. (1). In general, this condition fixes a broader or narrower range of possible solutions $f(g)$, none of which violates the positivity criterion. The positivity method described above converges towards a solution (within this range) the lowest value of which is as near as possible to zero.

$$
\min (f(g))=\max (0, \min (\tilde{f}(g)))
$$


By a slight modification, the method can also be made to converge towards a solution with

$$
\min (f(g)) \geq r, \quad 0 \leq r \leq 1
$$

In this case, the ODF can be split into a random part with volume fraction $r$ and a non-random part

$$
\left.f(g)=r \cdot{ }^{(r)} f(g)+(1-r) \cdot{ }^{(n r}\right) f(g), \quad{ }^{(r)} f(g) \equiv 1
$$

where ${ }^{(r)} f(g)$ and ${ }^{(n r)} f(g)$ are normalized in the usual way. Equation (22) expresses itself in terms of the coefficients

$$
C_{l}^{\mu v}=r \cdot{ }^{(r)} C_{l}^{\mu v}+(1-r) \cdot{ }^{(n r)} C_{l}^{\mu \nu}, \quad{ }^{(r)} C_{l}^{\mu v}=0 \text { for } l \neq 0
$$

from which follows for $l \neq 0$

$$
{ }^{(n r)} C_{l}^{\mu v}=\frac{C_{l}^{\mu v}}{1-r}
$$

The so defined function ${ }^{(n r)} f(g)$ fulfills the non-negativity condition Eq. (20) and its even coefficients are defined by Eq. (24). Hence, the positivity method described above can be applied to it. If a solution of the type Eq. $(21,22)$ really does exist, then the positivity method applied to $\left.{ }^{(n r}\right) f(g)$ converges to it. If no such solution exists, no convergence is reached. Hence, by varying the parameter $r$ in the range $0 \leq r \leq 1$ a range of possible odd functions $\tilde{f}(g)$ and hence of possible complete functions $f(g)$ can be obtained which all have the same pole figures (determined by the even part) and which do not violate the positivity criterion. Putting $r=0$ in Eq. (22), the solution according to Eq. (20) is obtained. If the pole figures are consistent with a real ODF, then this solution must necessarily exist. A solution with $r=1$ is only possible if all pole figures are random. In general, solutions with

$$
0 \leq r \leq R \leq 1
$$

will be possible. Thereby $R$ is a measure for the ambiguity range of the solution. The value $R$ can be estimated from pole figures. If the texture can be split into a random and a non-random part according to Eq. (22) then the same equation holds also for each pole figure:

$$
P_{h k l}(\alpha, \beta)=r \cdot{ }^{(r)} P_{h k l}(\alpha, \beta)+(1-r)^{(n r)} P_{h k l}(\alpha, \beta)
$$


If one assumes that the non-random ODF $\left.{ }^{(n r}\right) f(g)$ really assumes the value zero somewhere, then zero may be reached somewhere in the pole figures. It is however also possible that the lowest pole figure value is higher than zero

$$
\min \left({ }^{(n r)} P_{h k l}(\alpha, \beta)\right)=P_{\min } \geq 0
$$

Hence, Eq. (25) can be written more precisely

$$
0 \leq r \leq R \leq P_{\min } \leq 1
$$

It should be mentioned that the totality of all possible solutions $\tilde{f}(g)$ and hence $f(g)$ can be found by adding any odd function $\tilde{f}^{\prime}(g)$ to one of the above solutions such that

$$
-\min (\tilde{\tilde{f}}(g)) \leq R
$$

It must be further mentioned that for practical reasons the convergence of the positivity method can only be judged within the limits of error of the even function $\tilde{f}(g)$ which is obtained directly form the pole figures. This error usually consists of two parts, the experimental error in the measured pole figures and the mathematical error due to series truncation. Hence, the larger these errors the larger will be the practical ambiguity range $R$ of the solution.

\section{RESULTS}

In order to test the suggested procedure, one synthetic and two real textures were used. The synthetic texture was the superposition of a single component $\{001\}(100)$-texture possessing orthorhombic crystal and sample symmetries and a gaussian shape with a spread of $\omega_{0}=12.5^{\circ}$ and $50 \mathrm{vol} . \%$ of a random texture. The texture coefficients $C_{l}^{\mu \nu}\left(g_{0}, \omega_{0}\right)$ of a texture consisting of a single ideal orientation at $g_{0}$ with a gaussian spread $\omega_{0}$ are given by the expression

$$
C_{l}^{\mu \nu}\left(g_{0}, \omega_{0}\right)=\frac{\exp \left(-\frac{1}{4} l^{2} \omega_{0}^{2}\right)-\exp \left(-\frac{1}{4}(l+1)^{2} \omega_{0}^{2}\right)}{1-\exp \left(-\frac{1}{4} \omega_{0}^{2}\right)} i_{l}^{\mu v}(g)
$$

With $C_{l}^{\mu \nu}$ of Eq. (30) replacing ${ }^{(n r)} C_{l}^{\mu \nu}$ the coefficients of the total texture are obtained by Eq. (23) with $r=0.5$ and the texture itself is 


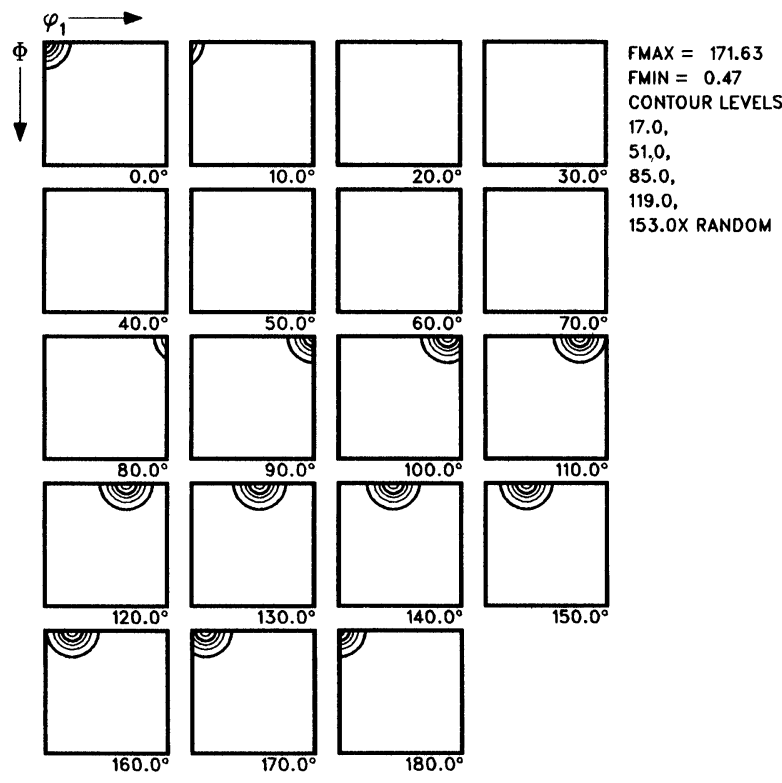

Figure 2 Complete ODF of an orthorhombic-orthorhombic $\{001\}\langle 100\rangle$-texture with $\omega_{0}=12.5^{\circ}, 50 \%$ superposed by a random texture $\left(L_{1}=23, \varphi_{2}=\right.$ const. $)$

calculated according to Eq. (5). Its graphical representation can be seen in Figure 2 for a degree of series expansion $L=23$, where the truncation error is small compared to the maximum value of the ODF. The minimum and maximum values of the ODF at $L=23$ can be seen in Table 2 compared to the values for $L=35$, where truncation errors can be neglected. The degree $L=23$ was chosen for economic reasons in calculation time.

Table 2 Minimum $f_{\min }$ and maximum $f_{\max }$ values of a synthetical texture for different degrees of series expansion $L$

\begin{tabular}{lll}
\hline & $f_{\min }$ & $f_{\max }$ \\
\hline$L=35$ & 0.500 & 172.21 \\
$L=23$ & 0.474 & 171.63 \\
\hline
\end{tabular}




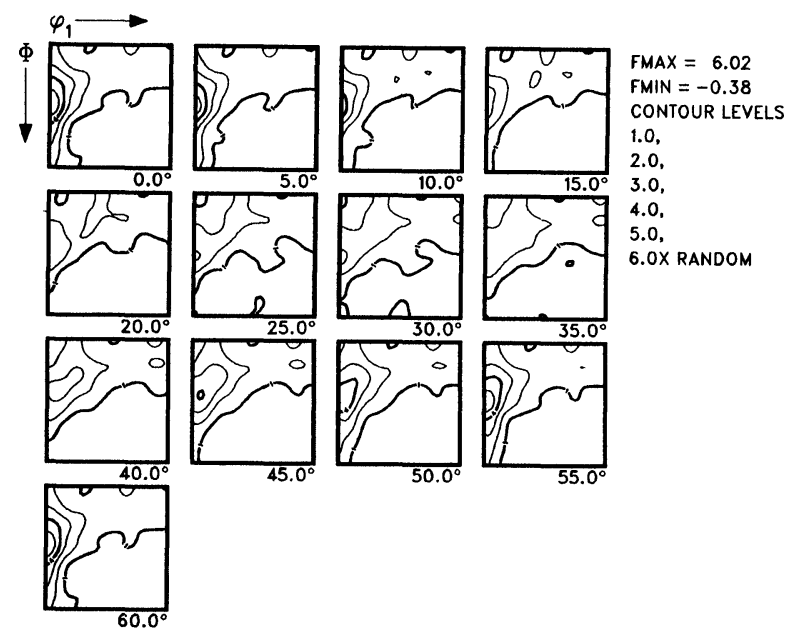

Figure 3 Even ODF of $80 \%$ rolled commercially pure titanium ( $L=22$, $\varphi_{2}=$ const.)

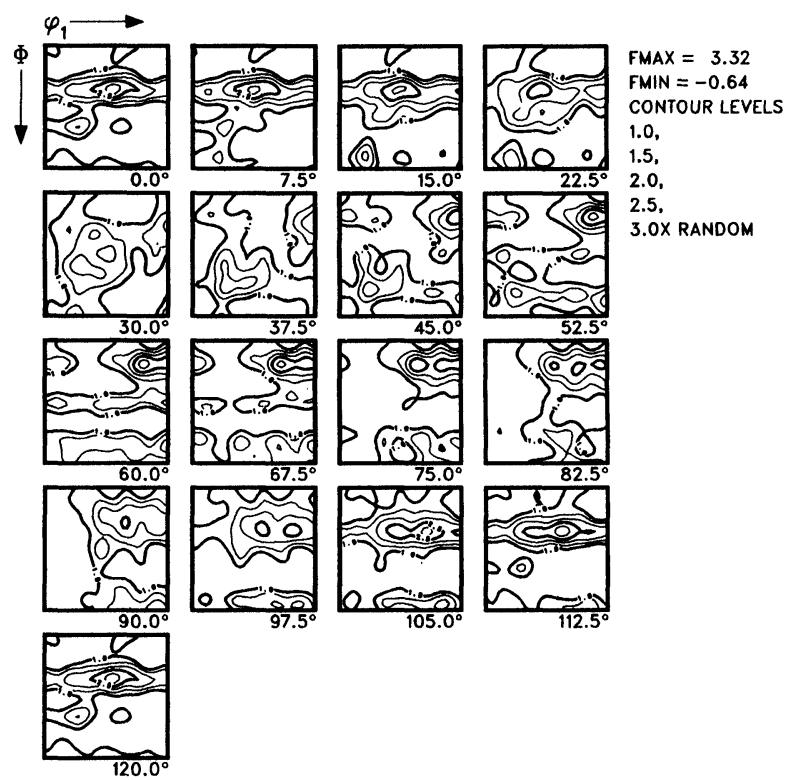

Figure 4 Even ODF of a sheared calcite specimen $\left(L=22, \varphi_{2}=\right.$ const. $)$ 
The real textures were the texture of $80 \%$ rolled commercially pure titanium (Schäfer et al. (1987)) and the texture of a sheared calcite specimen (Wenk et al. (1987)). In the case of titanium,

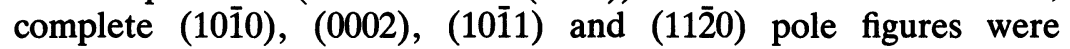
measured with neutrons in steps of $\Delta \alpha=5^{\circ}$ and $\Delta \beta=3.6^{\circ}$. The even order coefficients were calculated up to $L=22$ with additional

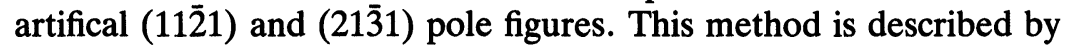
Dahms (1987) and Dahms \& Bunge (1987). For calcite, complete $(01 \overline{1} 2),(10 \overline{1} 4),(0006),(11 \overline{2} 0),(11 \overline{2} 3)$ and $(20 \overline{2} 2)$ pole figures were measured with neutrons in steps of $\Delta \alpha=\Delta \beta=5^{\circ}$. Here the even order coefficients were also calculated up to $L=22$ using artificial

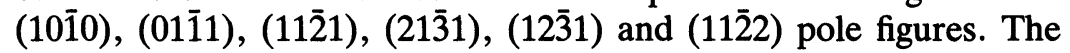
even ODF's of both textures can be seen in Figure 3 and Figure 4 in the projection $\varphi_{2}=$ const.

\section{Synthetic Texture}

The odd order coefficients were calculated using three assumed random texture components $r=0,0.17$ and 0.5 , respectively, up to $L=23$ for the iterative procedure. The iteration loop was run through 20 times. The second value 0.17 was chosen for a special reason which will be seen later. In Figure 5 , the change of $f_{\min }$ and the sum of negative intensities $S_{-}$during the iterative procedure is

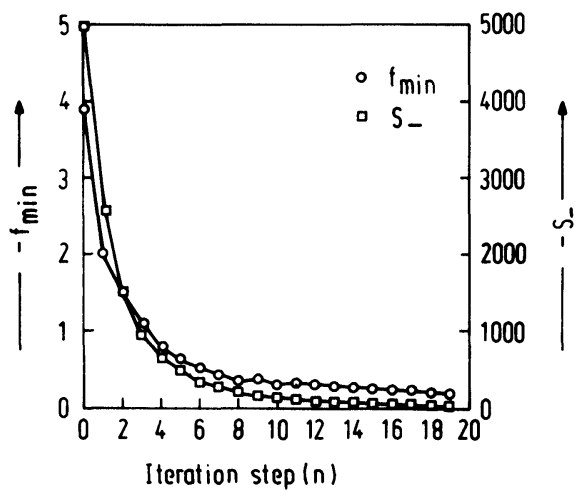

Figure 5 Change of the minimum value of the ODF $f_{\min }$ and the sum of negative intensities $S_{-}$of the synthetic texture during the iterative procedure. 


\begin{tabular}{|c|c|c|c|}
\hline$r$ & $f_{\min }$ & ${ }^{(n r)} f_{\min }$ & $f_{\max }$ \\
\hline & -0.1 & -0.1 & 141.64 \\
\hline. & & -0.2 & \\
\hline 0.5 & 0.460 & -0.080 & 170.81 \\
\hline theor. & 0.474 & - & 171.63 \\
\hline
\end{tabular}

shown for the case of $r=0$. It can be seen that $f_{\min }$ and $S_{-}$tend to reach zero, but the convergency is slow. In Table $3, f_{\min },{ }^{\left({ }^{n}\right)} f_{\min }$ and $f_{\max }$ of the resulting complete ODF can be seen for all assumed $r$-values compared to the theoretical values.

It is seen that for a known $r$-value, the iterative procedure leads to a good representation of the original data. On the other hand, it is obvious that a lower $r$-value may also lead to a set of coefficients which represents a positive ODF. In practice it is not possible to decide, which of the solutions $r=0.5$ or $r=0.17$ is physically more meaningful. Anyway it is useful to assume a certain value $r>0$, because then a positive ODF may be obtained by the iterative procedure. During the iteration, ${ }^{\left({ }^{n}\right)} f_{\min }$ approaches zero only after an infinite number of iteration steps.

\section{Texture of Titanium}

Here, the random texture component was chosen according to the minimum value of the measured (0002) pole figure $r=0.18$. According to the degree of series expansion in the even ODF $L=22, L_{1}=23$ was chosen for the odd ODF. The iteration was truncated after the 10th loop, when the absolute value of the minimum of the non-random texture component was less than half the $r$-value. The minimum value of the complete ODF was thus $f_{\min }=0.11$. The resulting complete ODF can be seen in Figure 6, a typical ODF for heavily rolled titanium with a maximum value $f_{\max }=6.39$ at $\left(0^{\circ}, 42.5^{\circ}, 0^{\circ}\right)$. 


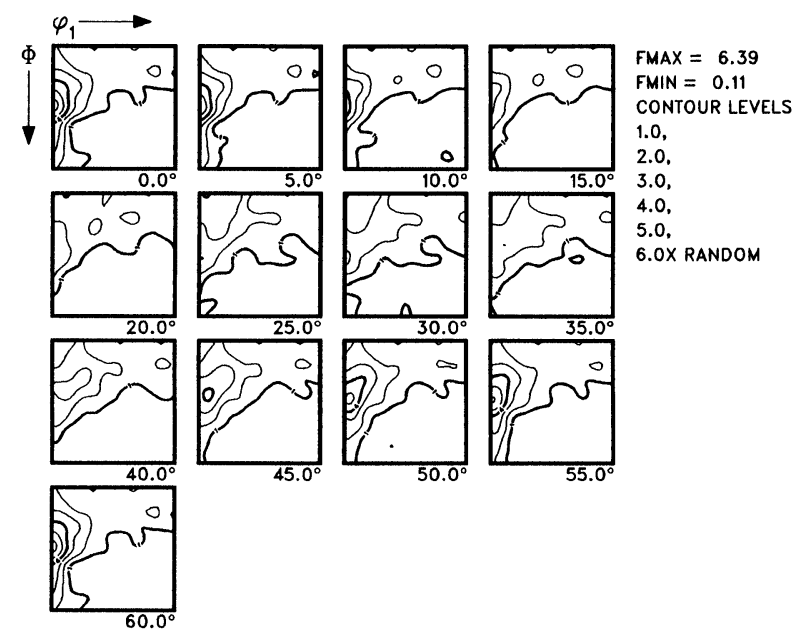

Figure 6 Complete ODF of the titanium specimen $\left(L_{1}=23, \varphi_{2}=\right.$ const. $)$

\section{Texture of Calcite}

In the case of the calcite specimen, the iterative procedure did not converge using the minimum of the measured (0006) pole figure as $r$-value. With $r=0.20$ and $L_{1}=23$, the iteration lead to a positive ODF within 6 min of calculation time. (A time-dependent truncation condition was chosen for economic reasons.) For the case of the calcite, the iterative procedure needs very much time, because

- the number of coefficients of a trigonal phase is more than double the number of a hexagonal one, and

- the region to be integrated in Euler space for a trigonal material is double the region for a hexagonal one.

The minimum value of the complete ODF was $f_{\min }=0.01$, which shows that the even order coefficients obtained from six measured pole figures together with also six unmeasured pole figures describe a physically possible texture. The resulting complete ODF can be seen in Figure 7 , with a maximum of $f_{\max }=4.34$ at $\left(65^{\circ}, 17.5^{\circ}, 67.5^{\circ}\right)$. This texture is not yet understood, but the present ODF might be a base for the understanding. 


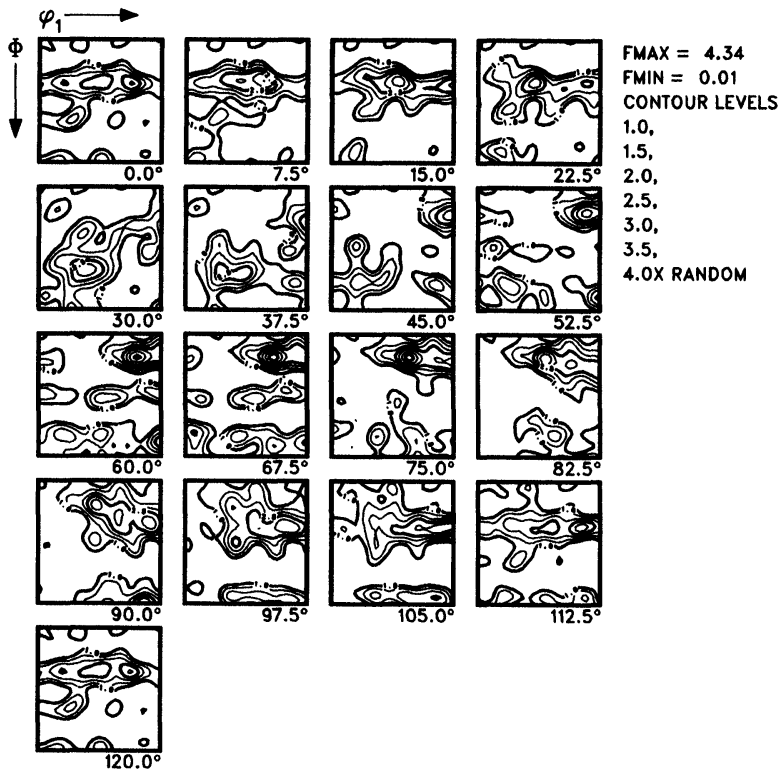

Figure 7 Complete ODF of the sheared calcite specimen $\left(L_{1}=23, \varphi_{2}=\right.$ const. $)$

\section{CONCLUSIONS}

An iterative method has been developed and tested, which allows to calculate odd order coefficients in the harmonic method. Only the physically necessary positivity condition for the complete ODF is introduced. The ambiguity of the solution is mathematically taken into account by introducing a random texture component into the calculations. This random texture component allows also to obtain positive ODF's after a finite number of iteration steps.

\section{References}

Bunge, H. J. (1982). Texture Analysis in Materials Science, Butterworth London.

Dahms, M. (1987). Spezielle mathematische Methoden der Texturanalyse und ihre Anwendungen unter besonderer Berücksichtigung der intermetallischen Phasen, Dr.-Ing. Thesis, TU Clausthal.

Dahms, M. \& Bunge, H. J. (Paper presented at the 8th ICOTOM, Santa Fe 1987) to be published by AIME. 
Lee, H. P. (1984). Texturanalyse unter Anwendung der Nullbereichsmethode, Dr.-Ing. Thesis, TU Clausthal.

Lee, H. P., Bunge, H. J. \& Esling, C. (1986). Textures and Microstructures 6, 289-313.

Lücke, K., Pospiech, J., Virnich, K. H. \& Jura, J. (1981). Acta Met. 29, 167-185.

Matthies, S. (1979). Phys. Stat. Sol. B 92, K135-138.

Schäfer, W., Höfler, S. \& Will, G. (1988). Textures and Microstructures 8, in press.

Van Houtte, P. (1983). Textures and Microstructures 6, 1-7.

Welch, P. I., Dahms, M. \& Bunge, H. J. (1987). In: Theoretical Methods of Texture Analysis, Ed. H. J. Bunge, DGM Informationsgesellschaft Oberursel 63-67.

Wenk, H. R., Kern, H., Pannetier, J., Höfler, S. \& Brokmeier, H.-G. (Paper presented at the 8th ICOTOM, Santa Fe 1987) to be published by AIME. 